\title{
A genetic analysis of the sex-determining gene, tra-1, in the nematode Caenorhabditis elegans
}

\author{
Jonathan Hodgkin \\ MRC Laboratory of Molecular Biology, Cambridge CB2 2QH, UK
}

\begin{abstract}
The normal sexes of Caenorhabditis elegans are the self-fertilizing hermaphrodite $(X X)$ and the male $(X O)$. The autosomal gene tra-1 is a major switch gene controlling sexual phenotype. Mutant phenotypes of 43 lossof-function (If) tra-1 alleles and 22 gain-of-function ( $g f$ tra-1 alleles are described and discussed. The tra-1(lf) alleles are recessive and, in general, masculinizing. The most severe mutations (such as seven out of eight identified amber alleles) can transform $X X$ animals into fertile males. These mutations have little effect on $X O$ animals (which are male already) but lead to some abnormalities in $X O$ gonadal development, indicating that tra-1 has functions in normal development of both sexes, although its major function is confined to the $X X$ hermaphrodite. Weaker tra-1(lf) alleles lead to incomplete masculinization of $X X$ animals, resulting in a variety of intersexual phenotypes. The tra-1(gf) alleles are dominant and have an opposite, feminizing effect. Six out of 22 can transform $X O$ animals into fertile females or hermaphrodites, whereas the remainder cause partial feminization. All 22 transform $X X$ animals into fertile females. Limited intragenic mapping indicates that the gene is large and that $g f$ alleles map to a location different from If alleles. The results suggest that the tra-1 gene has several roles in wild-type sexual development. First, tra-1 activity dictates female, as opposed to male, development in all nongonadal tissues of $X X$ animals. Second, tra-1 activity dictates female development in the somatic gonad of $X X$ animals. Third, a high level of tra-1 activity may act to inhibit spermatogenesis in the $X X$ germ line, thereby assisting the switch from spermatogenesis to oogenesis in the hermaphrodite. These three functions are all feminizing and specific to the $X X$ animal; the gene also has minor functions in the $X O$ animal, which are to assist normal male somatic gonad development and to promote abundant spermatogenesis in males. A low level of both spermatogenesis and oogenesis can occur in the absence of tra-1 activity. Both the function and the regulation of this gene must be complex.
\end{abstract}

[Key Words: Sex determination; nematode Caenorhabditis elegans; sex-determining genes]

Received May 6, 1987; revised version accepted July 21, 1987.

The genetic analysis of development in Caenorhabditis elegans has tended to focus on "switch genes," that is, loci with genetic properties indicating that they control important choices during development (Hodgkin 1984; Sternberg and Horvitz 1984). For example, the lin-14 gene appears to govern the relative timing of cell-division patterns in various tissues (Ambros and Horvitz 1987), and the lin-12 gene is involved in determining the spatial distribution of certain cell lineages (Greenwald et al. 1983). The analysis of sexual development has also yielded good examples of switch genes that control the choice between male and female pathways of development.

In normal development of $C$. elegans, animals with a single $X$ chromosome $(X O)$ develop into males, and animals with two $X$ chromosomes $(X X)$ develop into hermaphrodites, which are essentially modified females. Mutations in at least seven different autosomal genes can cause partial or complete alteration of the normal process, either masculinizing $X X$ animals or feminizing $X O$ animals. For one of these genes, tra-1, ("trans- former"), complete sexual transformations of both types have been demonstrated: loss-of-function $(1 f)$ alleles cause $X X$ animals to develop into fertile males, and gain-of-function $(g f)$ alleles cause $X O$ animals to develop into fertile females. As a result, it is possible to construct stable strains in which sex is controlled by the state of the tra-1 locus, not by $\mathrm{X}$ chromosome dosage (Hodgkin 1983b). This gene therefore appears to be a "master regulator" for sexual differentiation: tra-1 gene activity is both necessary and sufficient to promote female development, and lack of tra-1 activity is necessary and sufficient for male development.

The properties of the other six genes and the epistatic interactions between them and tra-1 suggested that their main wild-type role is to mediate between the primary sex-determining signal [X chromosome dosage or, more accurately, the $\mathrm{X}$ chromosome-to-autosome ratio (Madl and Herman 1979)] and the key gene tra-1 (Hodgkin 1980). In an $X O$ animal (X/A ratio low), these regulatory genes were proposed to render the tra-1 gene inactive ("OFF"), thereby permitting male development in both 
soma and germ line. In an $X X$ animal (X/A ratio high), tra-1 was proposed to be "ON" in the soma, promoting female development. In the $X X$ germ line, tra-1 would initially be OFF (permitting some spermatogenesis) but, later in development, would be ON (promoting oogenesis). The net result would be production of both sperm and oocytes in a female soma, resulting in a selffertile hermaphrodite phenotype. The regulatory interactions leading to these different activity states have been studied extensively at a genetic level, and the current model for the whole process is quite detailed (Hodgkin et al. 1985).

This paper reports an extensive mutational analysis of the tra-1 gene, based on the properties of $43 \mathrm{lf}$ (masculinizing) alleles and $22 g f$ (feminizing) alleles. The results indicate that correct tra-1 gene function is crucial to normal sexual development of all tissues and that this gene has a complex function. The simple model originally proposed $\left(X X: t^{t r a-1} \mathrm{ON}, X O: t r a-1 \mathrm{OFF}\right)$ is shown to require modification, because tra-1 appears to have a minor role in $X O$ animals, as well as its major role in $X X$ animals.

In characterizing these mutations, five main aspects of the sexual phenotype have been examined: germ line, somatic gonad anatomy, gross tail anatomy, yolk production, and overall fertility (either self-fertility, in the case of the hermaphrodite, or cross-fertility, in the case of males). The anatomical features are shown in Figure 1 .

\section{Results and discussion}

\section{Recessive (masculinizing) alleles}

Mutations of tra-1 were first recognized by the appearance of masculinized animals in mutagenized self-ferti- lizing hermaphrodite stocks $(X X)$. Some mutants appeared to be masculinized completely and were shown to carry and transmit two X chromosomes, thereby demonstrating that they were $X X$ animals transformed into fertile males (Hodgkin and Brenner 1977). General screens for a variety of C. elegans mutants have continued to yield recessive tra-1 alleles, to a total of seven to date.

Masculinizing mutations have also been deliberately sought by two methods: screening for extragenic suppressors of a gene her-1 (Hodgkin 1980) and by a general screen for mutations causing masculinization of $X X$ animals (see Materials and methods). Both of these methods yielded tra-1 mutants. This gene appears to be the only locus in C. elegans that can be mutated easily to yield fertile $X X$ males. Two related genes, tra-2 and tra-3, have also been identified, but tra-2 and tra-3 XX mutant males are masculinized incompletely and never fertile. The general screen for masculinizing mutations yielded several alleles of both tra-1 and tra-2, permitting an estimate of forward mutation frequency for these loci: about $2 \times 10^{-4}$ for tra-1 and $10^{-3}$ for tra-2. These mutations are therefore relatively frequent and likely to represent loss of function for the respective loci.

A large number of additional masculinizing alleles of tra-1 have been obtained by reverting a dominant, feminizing allele of tra-1. As explained in Materials and methods, this reversion was carried out in two steps: The strong dominant allele $e 1575$ was modified by the introduction of a linked modifying mutation, $e 1816$, and the double-mutant allele $e 1816 e 1575$ (which is still feminizing/ was used to select for cis-acting intragenic suppressors that would eliminate the dominant feminizing effect. A total of 27 recessive masculinizing alleles were obtained, ranging in strength from those causing
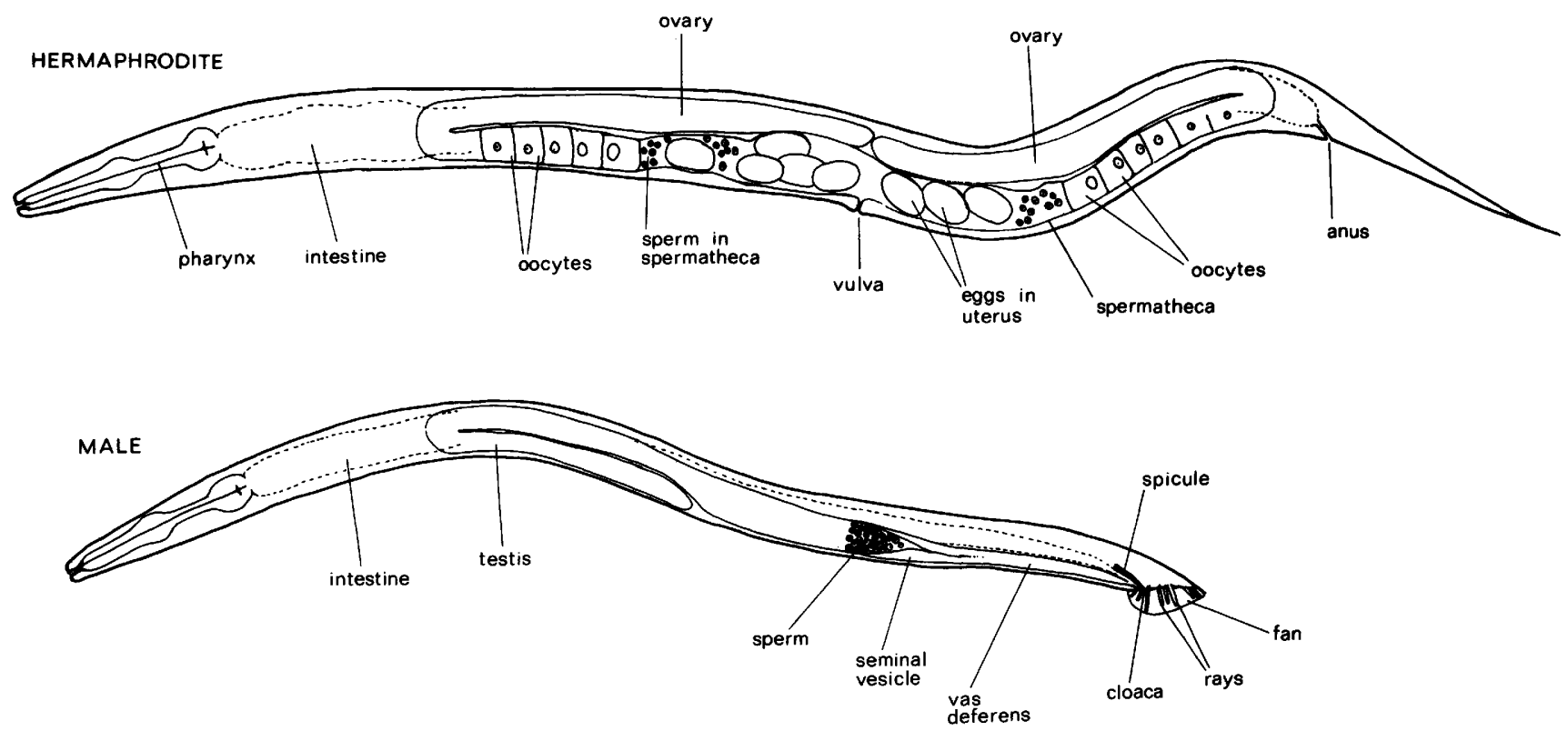

Figure 1. Major anatomical differences between hermaphrodite and male. The intestine of the adult hermaphrodite is specialized for the synthesis of large amounts of yolk proteins. 
complete $X X$ masculinization (the majority class) to those causing only slight masculinization. These alleles are distinguished from those isolated previously by the suffix $t r$, to indicate that they are triply mutant at the tra-1 locus. In most cases, the effects of the original dominant mutation are masked completely by the recessive masculinizing mutation.

One recessive lethal allele of tra-1 was obtained by this means, which is probably a small deficiency, as it fails to complement an essential gene adjoining tra-1 (see Materials and methods). A large tra-1 deficiency, $e D f 2$, has been described previously (Hodgkin 1980); this and the corresponding free duplication, $e D p 6$, have been used for varying gene dosage of tra-1 and for balancing tra-1 alleles, because $e D p 6$ carries tra-1 $1+1$.

\section{Null phenotype of tra-1}

Superficially, most recessive alleles of tra-1 appear to cause complete masculinization of $X X$ animals. All nongonadal structures examined so far have a male phenotype, behavior is indistinguishable from that of a wildtype male, and the $X X$ animals are capable of cross-fertilizing hermaphrodites. The complete transformation of sexual phenotype in the tail is illustrated in Figure 2B (tra-1 $X X$, indistinguishable from the wild-type $X O$ male tail illustrated in Fig. 2A). Weaker recessive alleles, which cause only partial masculinization, have also been obtained (see Fig. 2C-G). Stronger masculinization is seen when one of these alleles (e1076, Fig. 2F) is placed in trans with either $e D f 2$ or with the strong allele $e 1099$ (Fig. 3). For this reason, it appeared possible that $e 1099$ might represent a complete or almost complete loss of function.

For all of the strong alleles, $X X$ male fertility is much lower than that of wild-type $X O$ males, indicating that the germ line and/or somatic gonad is not normal in the tra-1 $X X$ males, as compared to wild-type $X O$ males. Gonadal phenotypes have been examined for a number of these strong alleles and found to fall into three main phenotypic classes (A1, A2, and A3 in Table 1). As discussed below, it is not certain which (if any) of these corresponds to a complete lack of tra-1 function, that is, a tra-1 null. Three alleles are described in detail, typifying each of the three classes: e1099, e1781, and e1835tr. Mutant gonads are illustrated in Figure 4, and histograms summarizing data are shown in Figure 5.

For tra-1(e1099) (class A1), the somatic gonadal phenotype is variable, ranging from a well-formed but undersized male gonad (Fig. 4A) to a disorganized mass (Fig. 4B). Oocytes or oocytelike cells are rarely seen in these gonads. In single male mating tests, 10/46 males were fertile, siring 2-262 progeny (mean 43). Wild-type $X O$ males are much more fertile: In one test, $6 / 6$ males were fertile, siring 213-2871 progeny (mean 1792).

In contrast, tra-1(e1781) $X X$ males (class A3) always have a large well-formed male somatic gonad, containing few or no sperm, and many large oocytelike cells (Fig. 4C). In mating tests, 3/36 males were fertile, siring 15-63 progeny (mean 27).

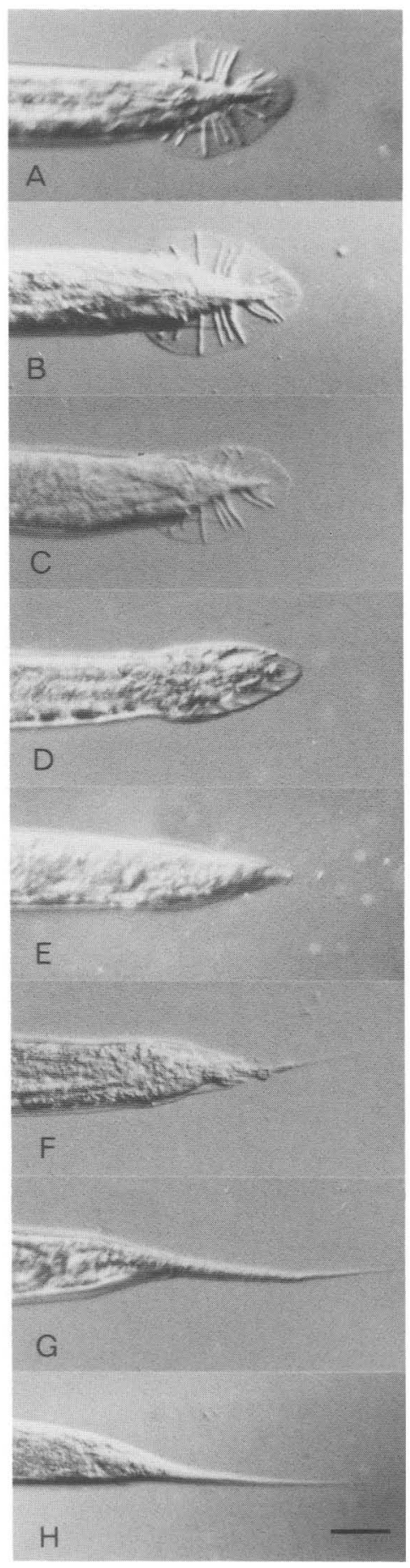

Figure 2. If mutants of tra-1: tail phenotypes, viewed ventrally. Bar, $25 \mu$. $(A)$ wild-type $X O$ (male); $(B-G)$ tra-1 $X X ;(H)$ wild-type $X X$ (hermaphrodite). The tra-1 alleles are $e 1099(B)$; e1488 (C); e1928 (D); e1929 (E); e1076 (F); e1732 (G) (see also Table 1).

The third allele, e1835tr (class A2), is intermediate between these two: Some animals resemble $e 1099$ in phenotype, but most resemble $e 1781$ (Fig. 4D). Fertility is higher than that for either of the other two alleles: $15 / 34$ 


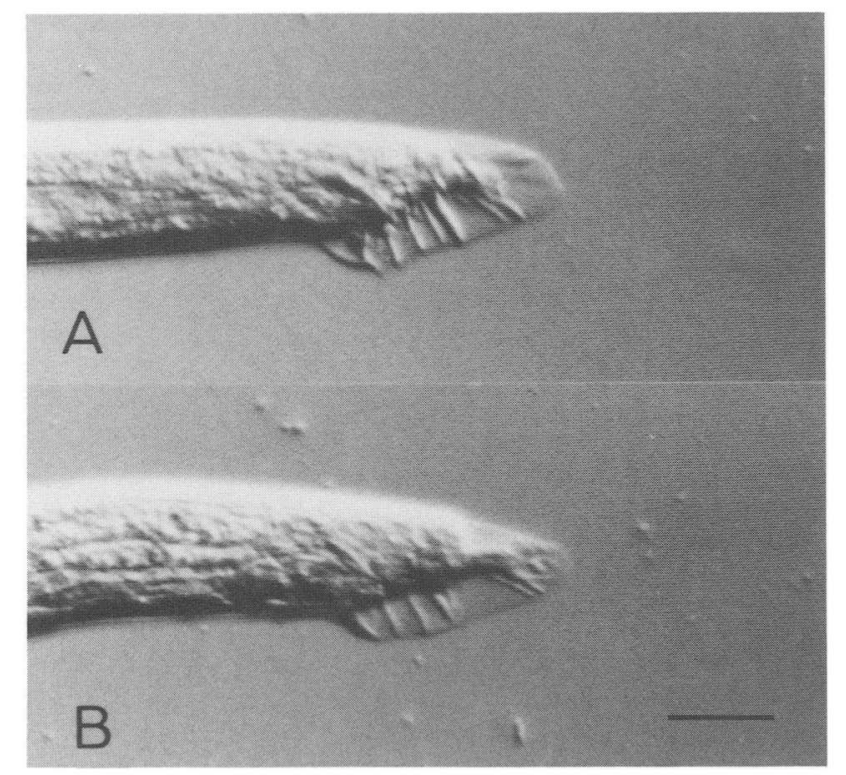

Figure 3. Tail phenotypes, semilateral view. Bar $25 \mu$. $|A|$ e1076/eDf2; (B) e1076/e1099 (cf. Fig. 2F).

males were fertile in mating tests, siring $1-125$ progeny (mean 44).

Thus, none of these tra-1 alleles, nor any of 10 other strong alleles examined, causes perfect masculinization of the gonad in an $X X$ animal: Either the soma or the germ line is abnormal. The division of these mutations into three classes, as in Table 1, is somewhat artificial, because there seems to be a spectrum of gonadal phenotypes in which the Al and A3 types represent the extremes and the $\mathrm{A} 2$ class includes all intermediates.

All of the strong alleles cause complete masculinization of tail structures and also of the intestine: None of those examined produces any yolk protein. It is likely this completely male nongonadal phenotype is the null phenotype of tra-1, for several reasons. First, strong alleles are found at gene knockout frequency (Greenwald and Horvitz 1980). Second, 37 alleles have been tested for suppressibility by the amber suppressor sup-7 (Waterston 1980), and eight amber alleles were identified. Seven of the eight are strong alleles, and the eighth is only slightly less masculinized than these (Hodgkin 1985). Third, as described above, strong alleles and the deficiency $e D f 2$ behave similarly in complementation tests (Fig. 3).

For the gonadal phenotype, matters are more complicated. The three types described above all occur fairly frequently. The availability of amber alleles does not provide useful information, because all three classes are represented among the amber alleles. The $e 1099$ allele is nonamber, and the $e 1781$ allele is amber. Seven of the triple alleles obtained by tra-1(gf) reversion are amber, and these can be compared with each other: Two resemble $e 1099$ (e1828tr and e1849tr), one resembles $e 1781$ (e1838tr), and four are intermediate $(e 1825 \mathrm{tr}$, e1835tr, e1844tr, and e1860tr).
Complementation tests using the eDf2 deficiency gave the results shown in Figure 5: $e 1099 / D F$ resembles $e 1099 / e 1099$, and $e 1781 / D f$ resembles e1781/e1781; however, e1781/e1099 resembles e1781/Df. This result makes it possible to argue that $e 1781$ does not correspond to the deficiency but that $e 1099$ does and, therefore, that e1099 is the "true null." Nevertheless, it is still possible to make alternative arguments in favor of either $e 1781$ or $e 1835$ tr being a true null.

It is unlikely that there is a completely different null phenotype such as lethality. First, although the set of amber alleles is heterogeneous, none of them affects viability, and most amber mutations in C. elegans are either null or close to null (Waterston 1980). Second, the experiments inducing If alleles by reverting the dominant $g f$ allele yielded no recessive lethal alleles when a point mutagen [ethyl methane sulfonate (EMS)] was used, but one lethal (e1855) was found when X-rays were used. This lethal fails to complement both tra-1 and an essential gene mapping close to tra-1, pha-1(e2123) ( $\mathrm{H}$. Schnabel, pers. commun.), so it is likely that $e 1855$ is a deficiency for both genes. Thus, lethals could have been recovered with EMS, but none was found. A dominant lethal null phenotype for tra-1 is excluded, because $e D f 2 /+$ is viable.

In conclusion, it is probable that the question of the true null will not be settled in the absence of molecular analysis, and maybe not even then. In dealing with a complex gene such as tra-1, this is perhaps not surprising.

\section{Recessive alleles: XO phenotype}

Initial experiments (Hodgkin and Brenner 1977) suggested that neither strong nor weak recessive tra-1 alleles had any effect on $X O$ animals, which appeared to be normal males. A test to compare the fertility of wildtype and tra-1 $X O$ males was carried out by selfing a hermaphrodite of genotype tra-1(e1099)/ + him-5 dpy-21. The him-5 mutation leads to the production of $X O$ self-progeny, and the $d p y-21$ mutation permits distinction between $X X$ and $X O$ animals, independent of sexual phenotype (Hodgkin et al. 1979; Hodgkin 1980). All $X O$ progeny were male; 36 individual males were picked and mated exhaustively with unc- 17 hermaphrodites, in order to measure maximum fertility (Hodgkin 1983a). Individual male genotypes were determined retrospectively, by progeny testing: $8 / 36$ were $+/ t$, siring 103-807 progeny; $16 / 36$ were tra-1/ + , siring 80-1006 progeny; 5/36 were tra-1/tra-1, siring $69-715$ progeny. Therefore, it appeared that at least some tra-1 XO males could exhibit high fertility, within the wild-type $X O$ male range. However, it was possible that fertility might be enhanced by the $d p y-21$ mutation; it was also possible that some tra-1 XO males might be completely infertile.

Therefore, tra-1 XO males of the three strong alleles described above were constructed explicitly (see Materials and methods), and gonadal phenotypes were examined by Nomarski microscopy. As shown in Figures 4 and 5 , these phenotypes are frequently abnormal, and in 
Table 1. XX phenotypes of tra-1(lf) alleles

\begin{tabular}{|c|c|c|}
\hline Class & Alleles ${ }^{a}$ & Phenotype \\
\hline Al & $\begin{array}{l}\text { e1099, e1728, e1729, e1783, e1828tr* } \\
\text { e18499tr* }\end{array}$ & fully male tail; somatic gonad small, variable, lacking oocytes; often fertile \\
\hline A2 & $e 1516, e 1835 t r^{*}, e 1844 t r^{*}, e 1860 t r^{*}$ & fully male tail; somatic gonad variable, often containing oocytes; often fertile \\
\hline A3 & $e 1781^{*}, e 1774, e 1838 t^{*}$ & $\begin{array}{l}\text { fully male tail; somatic gonad large, male, usually containing oocytes; } \\
\text { infrequently fertile }\end{array}$ \\
\hline A1-3 & $\begin{array}{l}\text { e1730, e1783, e1822tr, e1829tr } \\
\quad \text { e1832tr, e1833tr, e1834tr, e1836tr } \\
\text { e1845tr, e1856tr, e1857tr, e1858tr }\end{array}$ & fully male tail; gonad uncharacterized; sometimes fertile \\
\hline A4 & e440, e1766, e1830tr & fully male tail; gonad abnormal; infertile; fertile in trans with $e 1099$ \\
\hline A5 & $e 1825 t r^{*}, e 1850 t r$ & $\begin{array}{l}\text { slightly abnormal male tail (variable); somatic gonad variable, sometimes } \\
\text { containing oocytes; infertile; infertile in trans with } e 1099\end{array}$ \\
\hline A6 & e1928, e1847tr, e1859tr & $\begin{array}{l}\text { fan and rays reduced; somatic gonad usually male, containing sperm and } \\
\text { oocytes; never fertile }\end{array}$ \\
\hline A7 & e1929, e1837tr, e1840tr, e1853tr & fan and rays very reduced; gonad as A6 \\
\hline A8 & e1076, e1843tr & rays absent, fan almost absent, tail spike present; gonad as $\mathrm{A} 6$ \\
\hline A9 & e1732, e1846tr & almost fully hermaphrodite tail; gonad as A6; yolk present \\
\hline B1 & e1488 & fully male tail, variable hermaphrodite gonad, often self-fertile; yolk present \\
\hline B2 & e1764, e1831tr & $\begin{array}{l}\text { variable male tail; variable hermaphroditic gonad, some vulval induction; } \\
\text { never self-fertile; yolk present }\end{array}$ \\
\hline
\end{tabular}

a (*) Amber alleles.

general, $X X$ and $X O$ phenotypes are rather similar for each of these alleles. Some $e 1099 X O$ males have very abnormal gonads, like some $e 1099 X X$ males; others have a more normal male gonad, and oocytes have not been seen in e1099 XO males. However, apparent oocytes are seen in $X O$ males of both $e 1781$ and e1835tr. Male fertility for these alleles is low. Therefore, it appears that at least some aspect of tra-1 activity is required for full male fertility and probably for normal development of the male somatic gonad.

The requirement for tra-1 activity in the $X O$ seems to be low and almost dispensable. $X O$ male fertility has also been examined for a weaker tra-1 allele, e1488, and found to be similar to wild type.

\section{Weaker recessive alleles of tra-1: At least two allelic} series

As well as the strong alleles, which cause almost complete masculinization of $X X$ animals, a number of tra-1 mutations causing incomplete masculinization have been identified and studied. The phenotypes of these alleles are significant because they demonstrate that different aspects of the sexual phenotype can be varied independently and that tissues of opposite sexual phenotype can coexist in the same body.

Most of the weak alleles fall into one allelic series (Table 1, classes A4-A9). The weakest members of this series have a male (one-armed) somatic gonad, containing both sperm and oocytes, but the nongonadal anatomy is almost completely hermaphrodite (e.g., e1732, illustrated in Figs. 2G and 6A). Intermediate alleles show increasing levels of masculinization, as illustrated for the tail phenotype in Figure 2. Yolk protein production (Fig. 7) is detectable in mutants carrying the weakest alleles, but little or no yolk is made by any of the stronger mutants.

Several other recessive alleles of tra-1 have pheno- types that cannot be fitted into this allelic series (Table 1 , classes B1 and B2). The most remarkable is the $e 1488$ phenotype: $X X$ animals homozygous for $e 1488$ have a male tail anatomy and exhibit mating behavior, but the gonad (both soma and germ line) is essentially hermaphrodite (Figs. 2C and 6B). As a result, e1488 XX strains can be grown as homozygous self-fertile stocks. Only about half of the $X X$ animals produce self-progeny (31/51), and brood sizes are low: 1-28, mean 12. Eggs are never laid (in part, because the sex-specific musculature is male, so egg-laying muscles are absent), and consequently, the self-fertile hermaphrodites end up as "bags of worms" - empty carcasses surrounding their progeny, which eventually break free. The low self-fertility cannot be ascribed to the egg-laying defect, because other kinds of egg-laying defective mutants usually produce higher numbers. For example, six lin-3 bags were examined and found to contain 69-79 progeny each [lin-3 mutant hermaphrodites have defective vulval development [Sternberg and Horvitz 1984)]. Therefore, gonad function is not normal in the e1488 mutant. Vulval development also appears to be abnormal, because most $e 1488 X X$ individuals cannot be cross-fertilized by males. Cross progeny have been obtained from occasional animals.

Two other alleles, $e 1764$ and $e 1831$ tr, exhibit phenotypes similar to that of $e 1488$, as they often have hermaphroditic gonads and may exhibit partial vulval development. However, neither of these mutants is ever self-fertile. Both $e 1488$ and $e 1764$ make yolk proteins in abundance (Fig. 7). Another mutant that exhibits good male tail development but still makes yolk protein is the spontaneous allele $q 88$ (identified by T. Schedl, pers. commun.). In this mutant, the gonad has a male (A3) phenotype, and $q 88 X X$ males are often fertile.

It is possible that more detailed examination of tra-1 mutant phenotypes would reveal further complexities. 

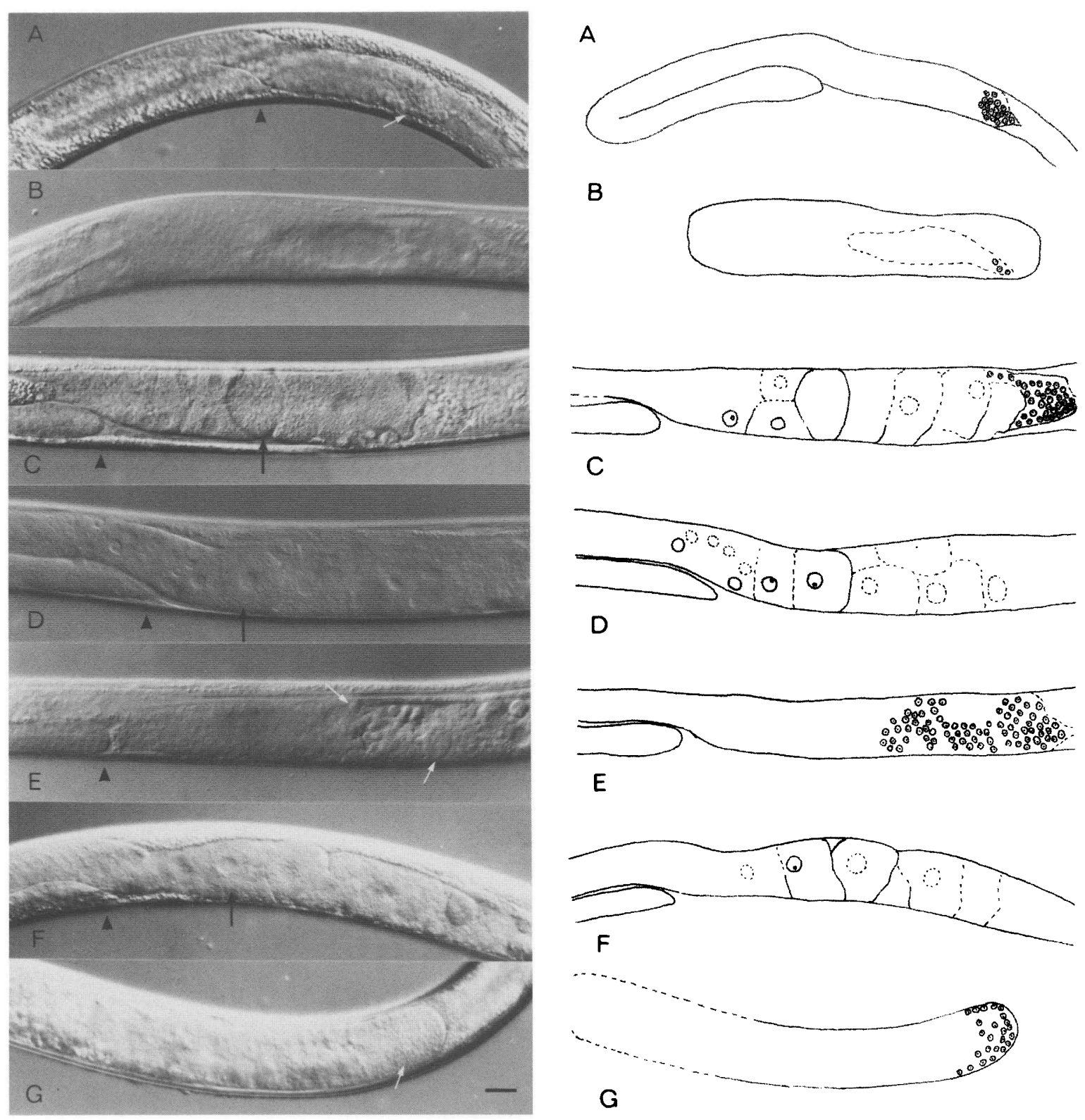

B
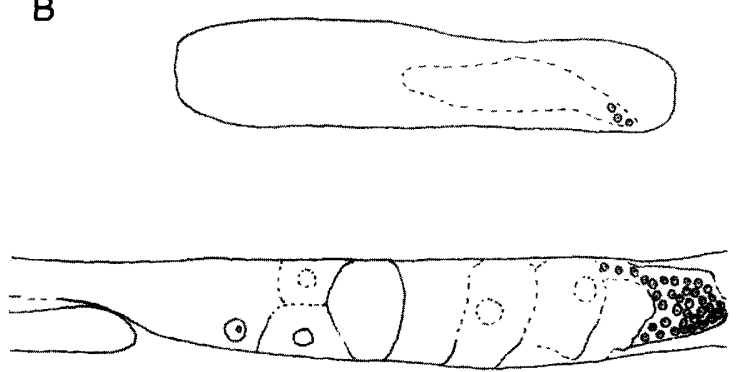

C
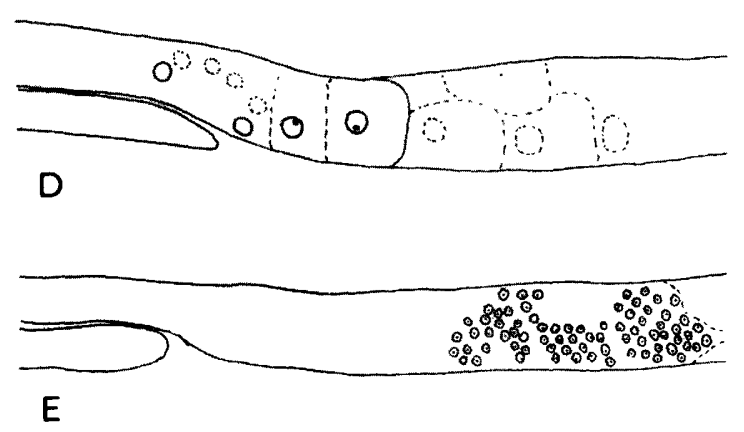

$E$

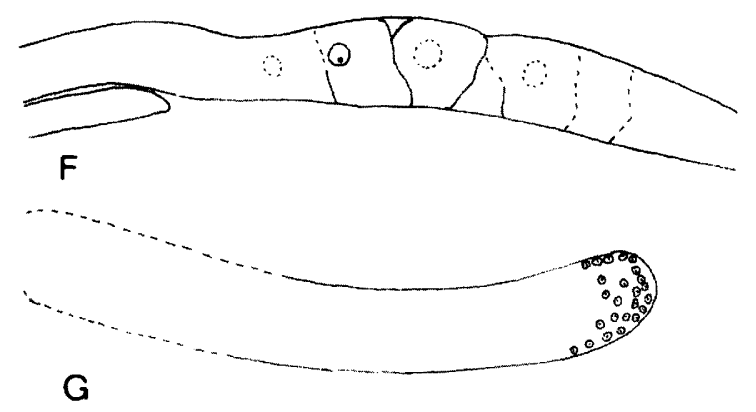

Figure 4. Gonadal phenotypes of wild-type and tra-1 mutants. (Left) Nomarski micrographs; (right) sketches indicating gonad outlines and gametes. Anterior is left, dorsal is up. Bar, $25 \mu$. White arrows mark sperm, black arrows mark oocytes, arrowheads mark the level of the distal tip of the gonad (see Fig. 1). (A) e1099 XX: small well-formed male gonad. (B) e1099 XX: abnormal ovoid vacuolated gonad. $(C)$ e1781 XX: male gonad containing sperm proximally, oocytes distally. $(D)$ e1835tr XX: male gonad containing oocytes. (E) lon-2 XO: wild-type male gonad containing sperm. $(F)$ e1781 1on-2 XO: male gonad containing oocytes. $(G)$ e1835tr lon-2 XO: abnormal ovoid gonad containing some sperm.

Already the data suggest that the tra-1 gene has a complex function: One class of partial mutant (A9) has a major effect on the gonad but little effect on the rest of the body, whereas another class (B1) has little effect on the gonad but a major effect on nongonadal anatomy. The two functions identified by these mutants are not wholly separable, because heterozygotes between mutations of the two classes exhibit an intermediate phenotype, rather than a wild-type phenotype. Heterozygous $e 1732 / e 1488 X X$ animals are variable and usually have an intersexual phenotype in both tail and gonad; few self-progeny are ever produced.

The mutant phenotype of alleles such as $e 1488$ and e1732 also suggests that sexual phenotype in different parts of the body is determined autonomously, rather than by a diffusible factor such as a hormone. Laser ablation experiments have already provided evidence for a great deal of cellular autonomy in sexual development 
(Sulston and White 1980), the only exception being the induction of vulval cell lineages, which is dependent on the somatic gonad (Kimble 1981).

\section{gf (dominant) alleles}

A total of $22 g f$ alleles have been isolated to date, using a variety of screens and selections (see Materials and methods). All cause dominant feminization, either partial or complete, in both $X X$ and $X O$ animals. All are tightly linked to the tra-1 locus, being less than 0.5 map units from recessive tra-1 alleles such as $e 1099$ or $e 1781$. This shows that they must be $g f$ mutations of some kind, because the deficiency $e D f 2$ has no dominant feminizing effect. The belief that all of these alleles are tra-1(gf) mutations rests on their map location and also on experiments demonstrating that $e 1575$, the first and best characterized of these alleles, is a cis-dominant tra-1 mutation. Selections for revertants of e1575 yielded recessive tra-1 masculinizing mutations, including seven amber alleles. One of these, e1838tr, has a phenotype closely resembling that of the amber allele e1781, and these two amber mutations may, in fact, be identical. This permits a cis-trans test: If the gf mutations were in a gene separate from the If mutations, a cis heterozygote $e 1575 e 1816 e 1838 /+++$ should have an identical phenotype to a trans heterozygote $e 1575 e 1816 / e 1781$. In fact, the latter genotype results in a female phenotype (both $X X$ and $X O$ ), whereas the former leads to a hermaphrodite $(X X)$ or male $(X O)$ phenotype. This must be because the cis-dominant effects

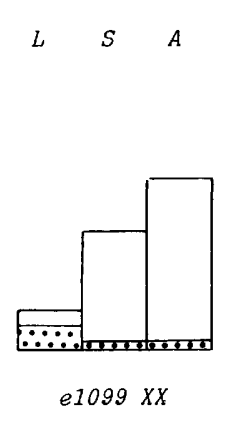

A

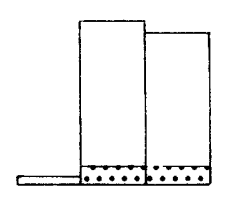

e1099/eDf2 XX

D

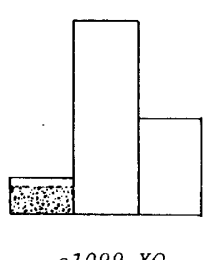

$\mathrm{G}$

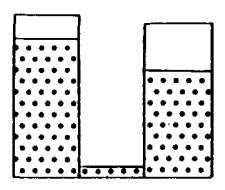

e1835XX

$\mathrm{B}$

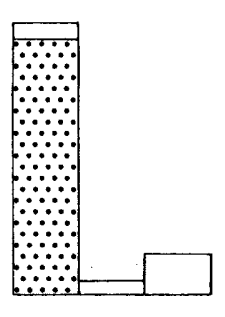

e1099/e1781 XX

E

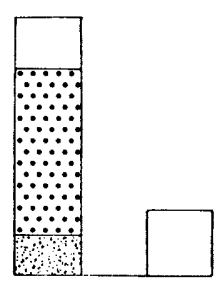

e1835 XO

$\mathrm{H}$

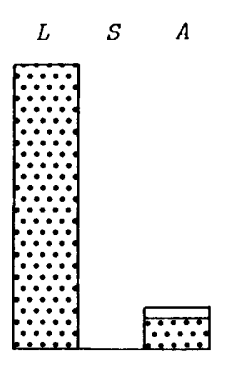

$e 1781 X X$

C

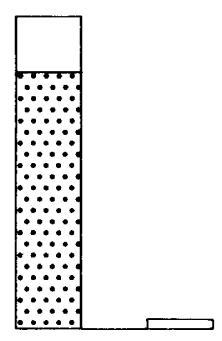

$e 1781 / e D f 2 X X$

$\mathrm{F}$

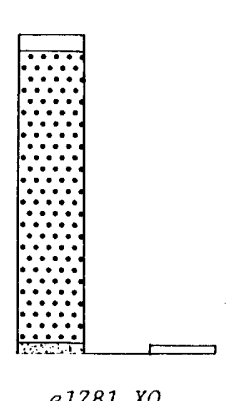

I
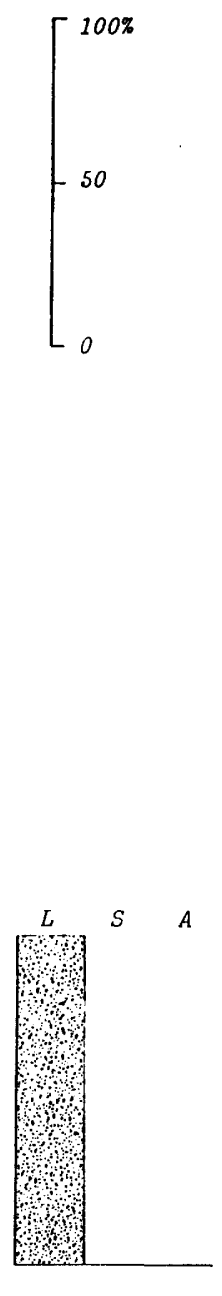

w. t. $X O$

$J$

Figure 5. Histograms summarizing gonad morphologies and gametogenesis, based on observations of 20-40 animals of each genotype. The bars show the proportions of animals with large, well-formed male gonads (L), small or thin well-formed gonads (S), and morphologically abnormal gonads (A); the last category includes a variety of anatomical derangements. The dotted region within each bar shows the proportion of gonads containing apparent oocytes; the stippled region shows the proportion with abundant spermatogenesis. $X O$ individuals $(G-I)$ were all hemizygous for lon-2, to distinguish them from $X X$ animals. For $G, H$, and $I$, most or all of the animals with abundant spermatogenesis (5-10\%) may have been $t$ ra-1/ + recombinants (see text and Materials and methods for further explanation). 


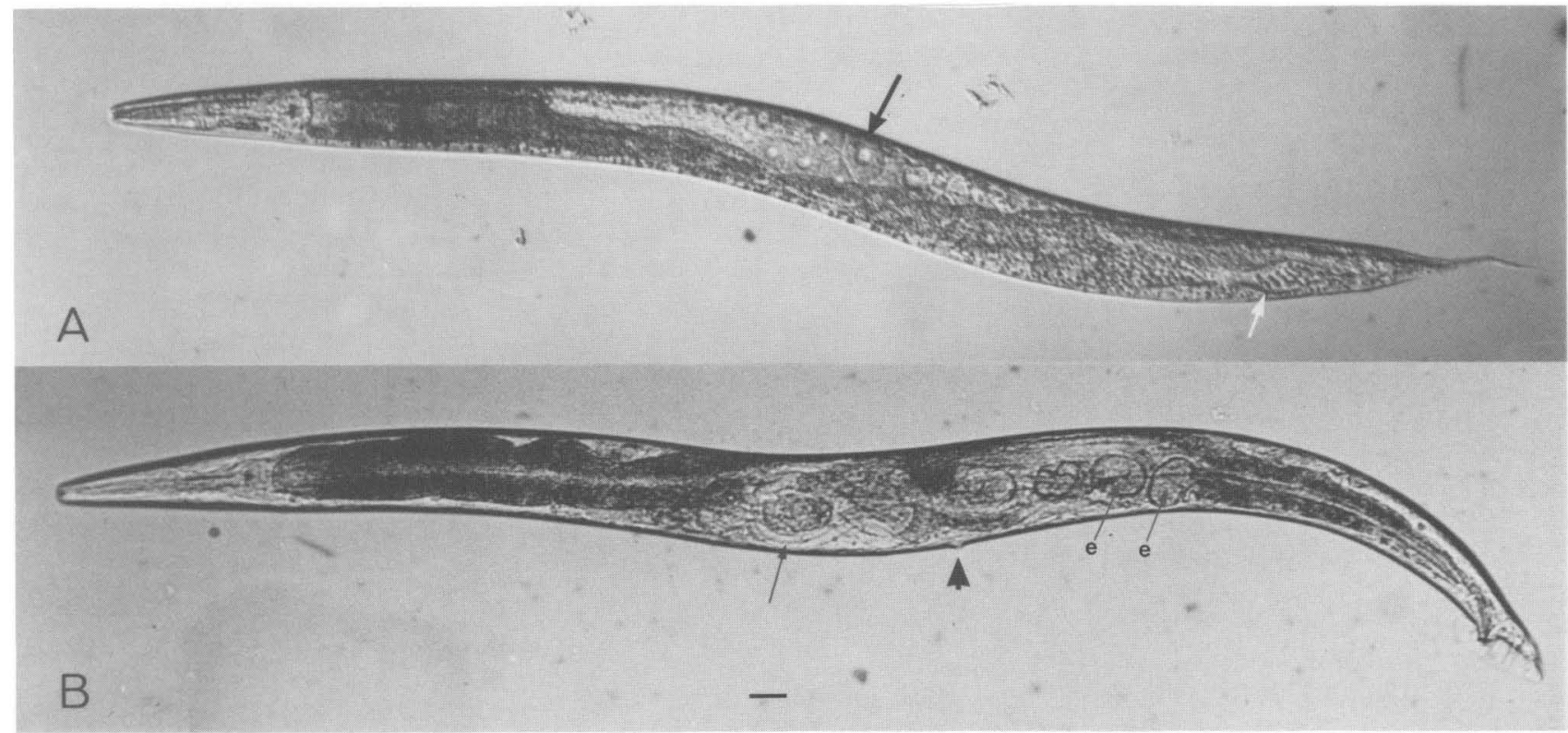

Figure 6. Weak If alleles of tra-1: bright-field photographs of whole worms. Bar, $25 \mu$. $(A)$ e1732 XX. $(B) e 1488 X X$. In $A$, note hermaphrodite tail and male somatic gonad, containing sperm trapped in a blocked vas deferens (white arrow) and oocytes more anteriorly (black arrow). In $B$, note male tail and eggs developing inside body (arrows marked e), as well as one hatched larva (single arrow); also rudimentary vulva (arrowhead).

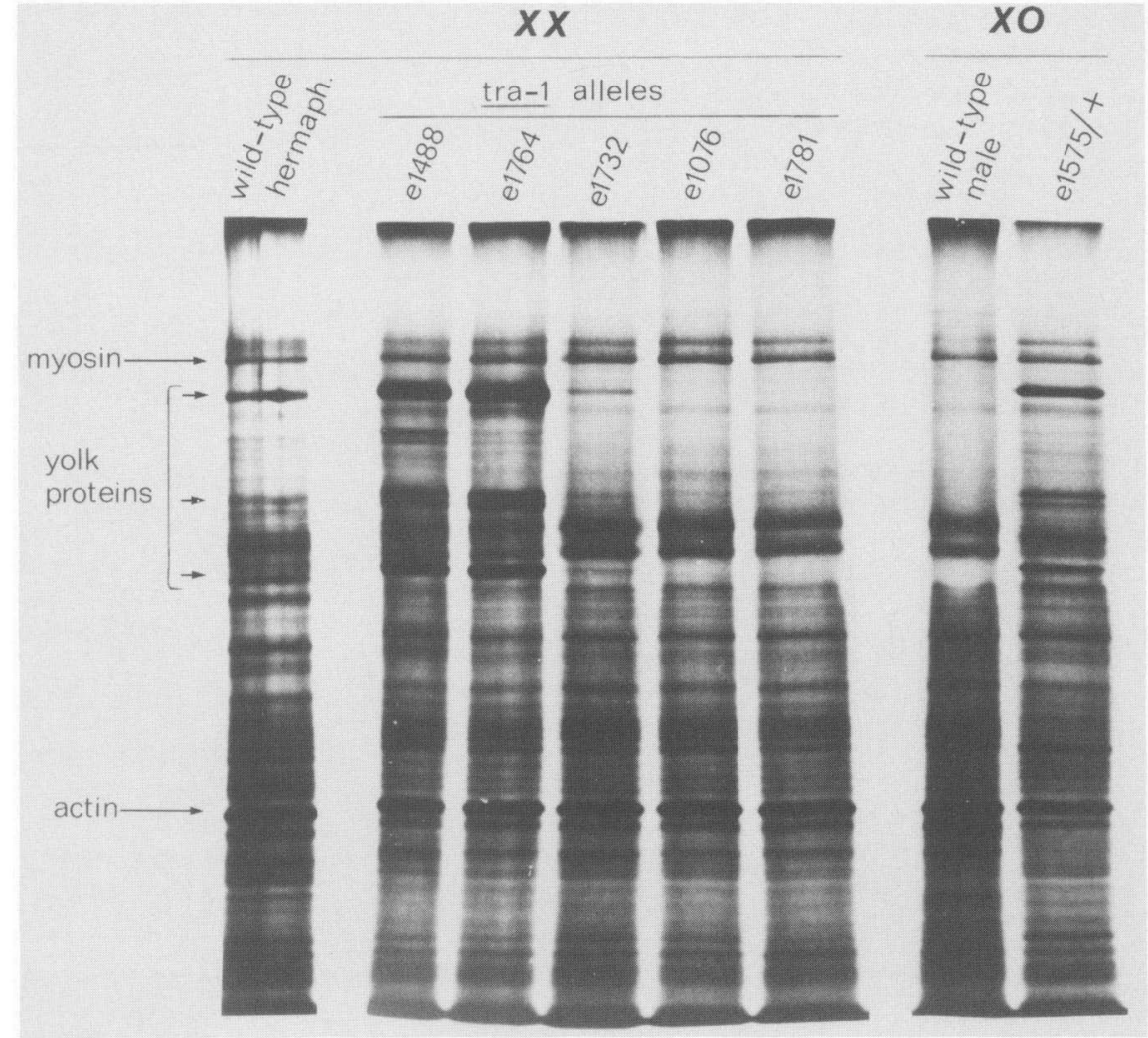

Figure 7. Yolk protein synthesis by wild-type and tra-1 mutant animals. Adults were labeled by feeding them on bacteria grown on $\left[{ }^{35}\right.$ S]sulfate media, and total proteins were analyzed by gel electrophoresis (Kimble and Sharrock 1983). 
Table 2. Dominant phenotypes of tra-1(gf) alleles

\begin{tabular}{|c|c|c|}
\hline Class & Alleles & Phenotype of $t r a-1(g f) /+X O$ \\
\hline 1 & $\begin{array}{l}\text { e1575, e1937, e1946, e2009, e2014, } \\
\text { e2109 }\end{array}$ & tail spike slightly truncated, gonad female, fertile (occasionally self-fertile) \\
\hline 2 & $e 1961, e 1962, e 2097$ & tail spike very truncated, gonad female, infertile (occasionally self-fertile) \\
\hline 3 & e1993, e1997, e1998, e2098, e2071 & tail intersexual, gonad female, abnormal vulval induction \\
\hline 4 & $e 1951, e 1953, e 2067, e 2198$ & $\begin{array}{l}\text { abnormal male tail, gonad intersexual, abnormal vulva; most animals rupture } \\
\text { at L4 molt }\end{array}$ \\
\hline 5 & $e 2013, e 2065, e 2069$ & abnormal, well-developed male tail, gonad intersexual, some vulval induction \\
\hline 6 & $e 2271$ & normal male soma, germ line differentiates both sperm and oocytes \\
\hline
\end{tabular}

of $e 1575$ have been abolished entirely by the amber mutation $e 1838$. The $e 1575$ lesion is demonstrably still present in the gene, because it can be recovered from the e1575e1816e1838 compound allele by intragenic recombination (see below). Also, suppression of $e 1838$ by the amber suppressor sup-7 restores dominant feminization, so that $e 1575 e 1816 e 1838 /+++$; sup- $7 / 0 \mathrm{XO}$ animals are intersexual rather than male. I conclude that $e 1575$ is a $g f$ allele of $t r a-1$. The other 21 gf alleles exhibit similar properties and map location to this allele, so I assume they are all tra-1 mutations.

Although all of these mutations cause dominant feminization, they differ in the strength of this phenotype. As indicated in Table 2, only six of the alleles lead to a fertile female phenotype in $\operatorname{tra}-1(\mathrm{gf}) /+X O$ heterozygotes. Crosses demonstrating the fertility of $e 1575 /+$ $X O$ animals have been reported previously; fertility is low, but this is a general characteristic of $X O$ females or hermaphrodites (Hodgkin 1986). Yolk protein synthesis in these animals is shown in Figure 7. The other 16 tra-1(gf) alleles lead to an intersexual or abnormal male phenotype. Some tail phenotypes for this allelic series are shown in Figure 8. The strength of the $X O$ phenotype is also correlated with the strength of the mutations as suppressors of tra-3 in $X X$ animals (Hodgkin 1986). For strong dominant alleles such as $e 1575$, tra- $1(g f) /+$, tra-3 $X X$ is female, but this genotype is hermaphrodite for weaker alleles. For very weak alleles, such as $e 2013$, this genotype results in a hermaphrodite phenotype with a masculinized (truncated) tail, indicating that the somatic masculinizing effects of tra-3 have been suppressed incompletely. The weakest of all these alleles, $e 2271$, is a recessive suppressor of tra-3 and, by itself, appears to cause feminization only of the germ line.

All but one of the $\operatorname{tra-1}(g f)$ alleles eliminate spermatogene is from tra-1(gf)/ $+X X$ animals, which therefore develop into fertile females. The exception is $e 2271$, for which $e 2271 /+X X$ animals are hermaphrodites of reduced self-fertility; homozygous $e 2271 X X$ animals are female (at $25^{\circ} \mathrm{C}$ ) or weakly self-fertile (at lower temperatures). None of the tra-1(gf) alleles appears to affect $X X$ somatic development, in tra-1(gf)/ +, tra-1(gf)/tra-1(lf), or (where tested) tra-1(gf)/tra-1(gf) genotypes. This indicates that the somatic functions of tra-1 in promoting female somatic development have not been altered. In

Figure 8. Tail phenotypes of $g f$ tra-1 alleles. All are $X O$ animals; $(A-C)$ viewed ventrally; $(D-H)$ viewed laterally. Bar, $25 \mu .(A)$ e1575/e1575; $(B)$ e1575/ $+; C)$ e1816e1575/ + ; $(D)$ e1575/e1099, $(E)$ e1993/+; $(F) e 1953 /+; G) e 2013 /+;(H)$ wild-type male.

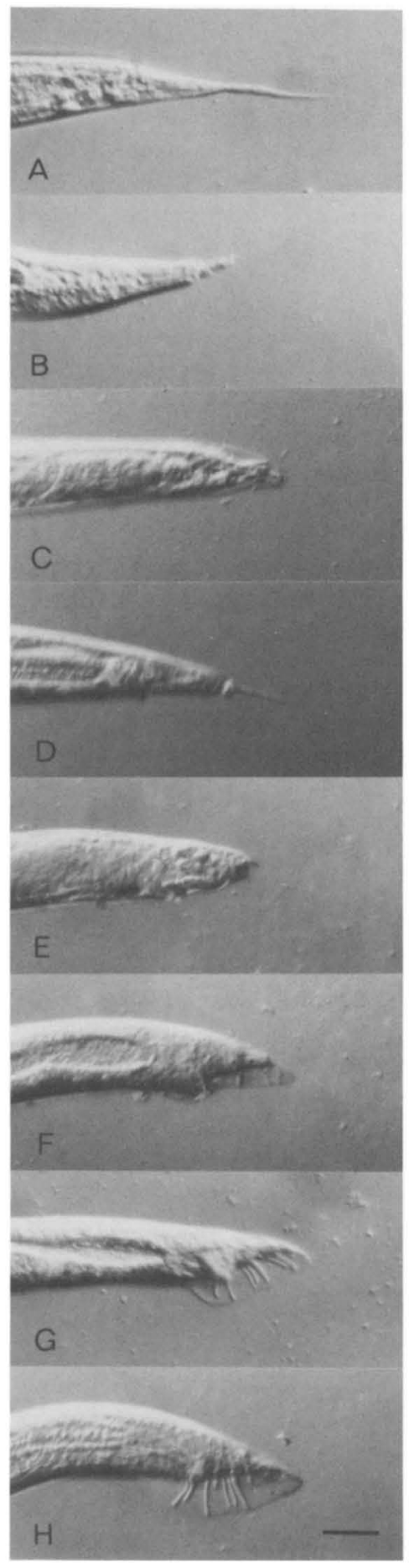


contrast, most tra-2(gf) alleles appear to be partly defective in the somatic functions of tra-2 (Doniach 1986). The tra-1(gf) mutations are therefore likely to be regulatory in nature. Nevertheless, it is conceivable that a tra-1 $(g f)$ phenotype might be caused by a truncated gene product. For this reason, 11 tra-1 $(g f)$ alleles were tested for suppression by the amber suppressor sup-7, with uniformly negative results.

\section{Dosage studies}

Gene dosage experiments were carried out to investigate further the nature of $\operatorname{tra-1}(g f)$ alleles. If these alleles result simply in an elevated level of tra-1 gene product, one would expect their phenotypic effects to be enhanced by additional copies of tra-1 $1+)$. Two aspects of the phenotype were examined: the self-fertility of $X X$ animals, and the tail phenotype of $X O$ animals.

As shown in Table 3, varying the dosage of the wildtype allele from one to three has no effect on $X X$ self-fertility. The large free duplication $e D p 6$, carrying tra-1(+), causes a reduction in fertility, but this seems to be due to a general effect on viability not caused by tra-1 itself. For two tra-1(gf) alleles, the feminizing effects (i.e., loss of sperm) are enhanced in tra-1(gf)/tra-1(lf) relative to $\operatorname{tra}-1(g f) /+$, which is the opposite of what is expected for a simple increase in the level of tra-1 gene product. It is more likely that these tra-1(gf) alleles are unresponsive to the normal control mechanism in the $X X$ animal. The fact that an additional wild-type allele acts in a masculinizing direction in this context is puzzling, though explicable by, for example, models in which the tra-1 gene is capable of autoregulation.

For the male tail phenotype, varying wild-type gene dosage from one to three has little effect. $X O$ males car-

Table 3. Effect of tra-1 gene dosage on XX self-fertility

\begin{tabular}{|c|c|c|c|}
\hline $\begin{array}{l}\text { tra-1 } \\
\text { genotype }\end{array}$ & $\begin{array}{l}\text { Dosage of } \\
\text { tra-1(+) }\end{array}$ & $\begin{array}{l}\text { Broods } \\
\text { counted }\end{array}$ & $\begin{array}{l}\text { Average } \\
\text { number of } \\
\text { progeny }^{\mathbf{a}}\end{array}$ \\
\hline$+/+$ (wild type) & 2 & 12 & 329 \\
\hline$+/ e D f 2$ & 1 & 7 & 219 \\
\hline +/e1099 & 1 & 4 & 356 \\
\hline$+/ e 1076$ & 1 & 4 & 355 \\
\hline$+/+; e D p 6$ & 3 & 6 & 263 \\
\hline$+/ e D f 2 ; e D p 6$ & 2 & 8 & 339 \\
\hline$e 1099 / e D f 2 ; e D p 6$ & 1 & 6 & 285 \\
\hline e1099/e1099; eDp6 & 1 & 6 & 253 \\
\hline$e 1575 /+$ & $1+g f$ & 12 & 0 \\
\hline e1816e1575/+ & $1+g f$ & 24 & 6 \\
\hline e1816e1575/e1099 & $0+g f$ & 12 & 0 \\
\hline$e 1816 e 1575 / e 1781$ & $0+g f$ & 30 & 0 \\
\hline$\left(15^{\circ} \mathrm{C}\right)$ & 2 & 6 & 336 \\
\hline$e 1816 e 1575 /+$ & $1+g f$ & 12 & 77 \\
\hline$e 1816 e 1575 / e 1099\left(15^{\circ} \mathrm{C}\right)$ & $0+g f$ & 12 & 1 \\
\hline$e 1816 e 1575 / e 1781\left(15^{\circ} \mathrm{C}\right)$ & $0+g f$ & 30 & 1 \\
\hline$e 2013 /+$ & $1+g f$ & 15 & 45 \\
\hline$e 2013 / e 1099$ & $0+g f$ & 15 & 0 \\
\hline$e 2013 / e 1781$ & $0+g f$ & 15 & 0 \\
\hline
\end{tabular}

a Progeny counts included both viable progeny and unhatched eggs. rying $e D p 6$ [i.e., three copies of $t r a-1(+)$ ] are of low fertility, but again, this is probably a nonspecific effect caused by the large size of this duplication, and there is no sign of anatomical derangement that could be ascribed to excess tra-1 activity (data not shown). For the $g f$ allele $e 1575$, tail phenotypes are similar in tra-1 $(g f) /+$ and tra-1(gf)/tra-1(lf) $X O$ animals (Fig. 8B,D). In one dose, $e 1575$ does not cause complete feminization of the tail, which carries a truncated tail spike. In two doses, a complete tail spike is made (Fig. 8A), indicating that this allele is capable of causing a complete transformation of an $X O$ animal. None of the other tra-1(gf) alleles isolated so far is any stronger, indicating that none of them results in as high a level of tra-1 activity as is found in an $X X$ animal, a her- $1 X O$ animal, or a fem $X O$ animal (Hodgkin 1986). In all of these genotypes, a single copy of tra-1(+) is sufficient for a perfect female tail spike; therefore, it appears that none of the tra-1(gf) alleles is fully deregulated. It may be that a very complex mutation would be required to achieve complete deregulation; alternatively, such a mutation might have damaging pleiotropic effects. There is some reason to believe that premature tra-1 expression could have damaging consequences during embryogenesis (Hodgkin 1986).

In conclusion, it appears that the $g f$ allele $e 1575$ does not cause overproduction of tra-1 product but, instead, renders the gene (or its product) insensitive to the normal regulatory mechanism, presumably mediated by the negative action of $\mathrm{fem}$ gene products (Hodgkin 1986). Consequently, the tra-1 product remains at a high level of activity in both $X X$ and $X O$ animals, resulting in feminization in both cases. This allele can therefore be regarded as a "constitutive" for tra-1 activity, rather than an "overproducer." Other gf alleles have not been analyzed to the same extent as $e 1575$, but there is no reason to believe that they would behave very differently. Genetic observations such as these do not indicate whether the inappropriate regulation of tra-1 in these mutants occurs at DNA, RNA, or protein levels.

\section{Intragenic mapping}

A limited set of mapping experiments, using a variety of protocols, have yielded the following results. First, the $g f$ allele $e 1575$ maps to the right of all eight independently isolated amber alleles and of other recessive alleles. Second, approximate intragenic map distances vary from 0.02 map units for the distance e1828-e1575, to 0.15 map units for $e 1825-e 1575$. Third, the modifying mutation $e 1816$ maps either very close to $e 1825$ or further to the left. Fourth, five $g f$ alleles all map to the right of $e 1099$ and, in one case, to the right of $e 1575$. In sum, the gene is large, and the structural part of the gene (identified by the amber alleles) is probably separate from the regulatory part of the gene. A map of the tra-1 region is shown in Figure 9A, and a preliminary intragenic map of tra-1, in Figure 9B.

The most informative experiments involved dissection of the triply mutant alleles such as e1825tr, that is, e1816e1825e1575. From a heterozygote vab-7 + dpy$18 /$ + tra-1(e1825tr $)+, 134 \mathrm{Vab}$ recombinants were 
A


B

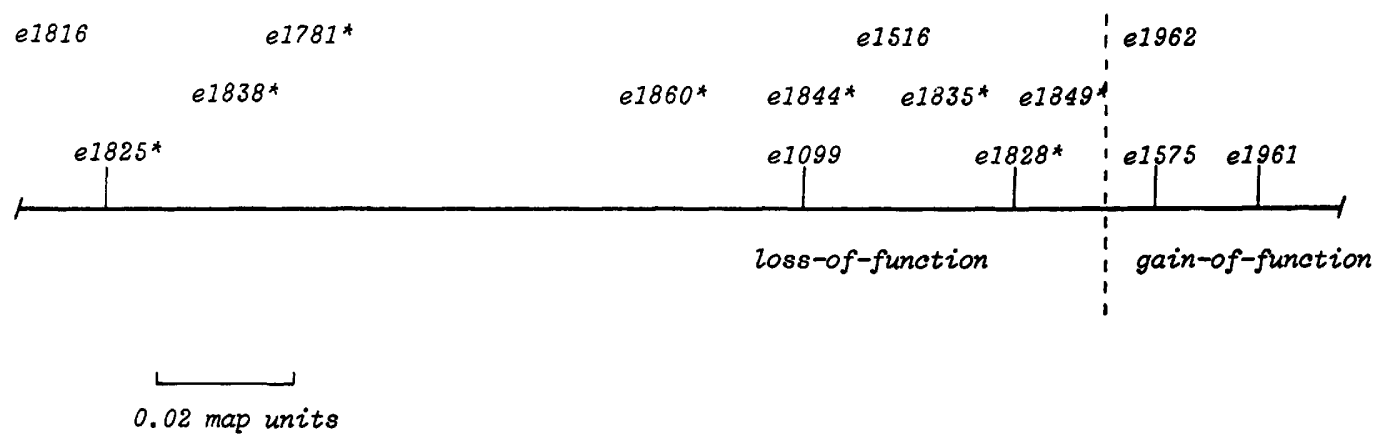

Figure 9. Genetic maps of tra-1 region and fine structure of tra-1, showing markers used in this study. $(A)$ The region shown is about one-quarter of LGIII; unc-32 is centrally located on LGIII. Both $e D f 2$ and $e D p 6$ extend to the right-hand end of LGIII. The gene pha-1 was mapped by H. Schnabel (pers. commun.). For a more complete map of LGIII, see Swanson et al. (1984). (B) Rough intragenic map of tra-1; scale expanded 67-fold relative to $A .\left(^{*}\right)$ Amber alleles. The order and spacing of the alleles above the line is tentative, because it is based on few events and on estimates of recombination frequency, rather than exact measurements.

picked: 65 carried vab-7 e1825tr, 8 carried vab-7 e1575, and 61 carried vab-7 +. Also picked were 92 Dpy recombinants: 46 carried $+d p y-18$, and 46 carried e1825tr $d p y-18$. The two-factor distance between $v a b-7$ and dpy-18 was also measured in this experiment and found to be 2.5 map units. Thus, e1825 maps to the left of e1575, and the distance between the two mutations is $8 / 134 \times 2.5$, that is, about 0.15 map units. In contrast, in analogous crosses using a different amber allele (e1828tr) only $1 / 68$ recombinants in the tra-1-dpy-18 interval carried tra-1(gf), showing that $e 1828$ is much closer to $e 1575$. One may speculate that the $e 1825 \mathrm{mu}$ tation, which clearly has residual tra-1 function, lies close to the $3^{\prime}$ end of the coding portion of tra-1.

Dissection of the triple alleles was also achieved by constructing strains of genotype unc-32 vab-7 + dpy18/unc-32 + tra-1|tr) + . In the Unc-32 phenotypic background, females [carrying tra-1(gf)] are much more conspicuous than those in a wild-type phenotypic background and can be detected in large populations $\left(10^{3}-10^{4}\right.$ animals per plate). Using this semiquantitative method, seven amber alleles were shown to lie to the left of $e 1575$. The allele $e 1825$ recombined most readily of the set (14 events recovered), consistent with the previous result; the allele $e 1838$ also recombined readily. Out of a total of 22 recombinants for $e 1825$ and 21 for the other six alleles, all carried e1575 rather than e1816e1575. Consequently, the $e 1816$ modifying mutation must lie well to the left of $e 1575$, and probably left of $e 1825$.

An estimate for the distance from $e 1099$ to $e 1575$ was obtained by picking non-Unc Tra(lf) recombinants from hermaphrodites of genotype unc-47 tra-1(e1099)/ +tra1(e1816e1575); tra-3(e1107). These recombinants may be either Tra-1(lf) or Tra-3(lf), which are distinguishable, but a Tra-3 phenotype can only be seen in a tra-1 $1+1$ background, because both tra-1(lf) and tra-1(gf) mutations are epistatic to tra-3 (Hodgkin 1980). Of these, $3 / 327$ were Tra-3 and 324/327 were Tra-1, giving a distance of about one hundredth of the distance from unc- 47 to tra-1, that is, 0.05 map units.

To determine the relative order of other dominant and recessive tra-1 alleles, a different protocol was used. Male/female strains consisting of tra-1(lf) males and tra-1(gf)/tra-1(lf) females can be stably propagated for several generations (Hodgkin 1983b), but if the two mutations are recombinationally separable, a tra $-1(+)$ allele 
will eventually be generated. This will result in the appearance of hermaphrodites that will take over the population rapidly. Inclusion of linked outside markers (unc-32, dpy-18, or sup-1) on either of the two original chromosomes permits relative ordering. Establishment of male/female strains of this type was used routinely to demonstrate tight linkage of all tra-1(gf) alleles to tra-1(e1099), and 10 of these strains were maintained for 4-34 additional generations. All eventually produced a tra- $1(+\mid$ recombinant, usually within the first 10 generations (representing $<10^{5}$ individuals). In four cases where an outside marker was included, the tra-1(gf) allele was shown to lie to the right of tra-1(e1099) (alleles $e 1951, e 2009$, e2013, and e2014). In initial experiments, the recessive alleles $e 1099, e 1516$, and $e 1781$ were shown to lie to the left of $e 1575$, the reference $g f$ allele.

One exception to the rule that tra-1 recessive alleles are recombinationally separable from tra-1(gf) alleles is the unusual allele $e 1488$. A male/female strain $e 1488 /$ e1575; him-5 was constructed and grown for over 350 generations, without yielding a tra-1 $(+)$ recombinant. Eventually one did appear, at approximately generation 400 , but it is clear that $e 1488$ and $e 1575$ must be extremely tightly linked. The $e 1488$ mutation does not appear to interfere with recombination, because recombination between the flanking markers unc- 47 and $d p y-18$ was found to be the same in trans with both tra-1 $1+1$ and tra-1(e1488).

Male/female strains can also be used to test for recombination between different tra-1(gf) alleles, by using a triple allele as the tra-1(lf) allele. For example, a strain of tra-1(e1828tr) males and tra-1(e1961)/tra-1(e1828tr) females was established but yielded a tra-1 $+\mid$ recombinant after 12 generations, indicating that $e 1575$ and $e 1961$ are separable, with $e 1961$ probably to the right (assuming that this was not a gene conversion event). However, another strain of the same type, using $e 1962$ as the tra-1(gf) allele, failed to yield a tra-1 $1+1$ recombinant after more than 50 generations, suggesting that $e 1962$ lies to the left of $e 1575$ or is inseparable from it.

These mapping experiments indicate that the total recombinational length of the tra-1 gene is at least 0.15 map units. This is much larger than the lengths estimated for two large muscle genes, unc-22 and unc-54, which are less than 0.04 map units in extent (Moerman and Baillie 1979; Waterston et al. 1982). Either tra-1 is a very big gene, or it experiences considerable map expansion. The relationship between physical distance and recombinational distance in the C. elegans genome is known to vary considerably, from less than $300 \mathrm{~kb} / \mathrm{map}$ unit to at least $900 \mathrm{~kb} / \mathrm{map}$ unit (Greenwald et al. 1987).

\section{Conclusion}

The main conclusions from this study are that activity of the tra-1 gene is the major factor controlling the sexual phenotype of the soma and that it also plays a significant role in controlling germ-line phenotype. The gene appears to be large, at least in terms of recombinational map distance, and to have a complex function.
As far as nongonadal aspects of sexual phenotype are concerned, the role of tra-1 appears to be simple: Activity directs female development, and inactivity directs male development. This follows from the observations that most tra-1(lf) alleles result in completely male development of the tail, intestine, and so on, in both $X X$ and $X O$; therefore, tra-1 activity must be necessary for normal female somatic development in wild-type $X X$ animals. Furthermore, tra-1 activity is also sufficient to direct female development in $X O$ animals.

The weaker alleles of tra-1 (classes A4-A9, B1-B2) have properties suggesting that tra-1 has a complex function in the nongonadal parts of the soma, because mutants have phenotypes consisting of mixtures of male, female, or intersexual tissues. Some of these phenotypes may result from threshold effects: A partly defective tra-1 allele may have sufficient activity to direct female development in one tissue but not in another, which therefore develops in a male or intersexual pattern. Threshold effects cannot explain why the mutants do not all fall into one allelic series, so it is likely that there is more than one component to tra-1 function. Class A and class B mutants do not fully complement each other, suggesting that these components are partly overlapping. Ambros and Horvitz (1987) have found similar behavior in the heterochronic lin-14 gene. Exhaustive characterization of mutant phenotypes would probably subdivide these classes further but would require a great deal of work, in view of the dozens of sexually dimorphic features that have been documented so far (Sulston and Horvitz 1977; Kimble and Hirsh 1979; Sulston et al. 1980).

The role of tra-1 in the somatic gonad is more complicated. Female gonadal development requires tra-1 activity, but some part of tra-1 activity is also required for completely normal male gonadal development. This is inferred from the fact that many tra-1(lf) alleles (classes A1 and A2) lead to a variably abnormal somatic gonad in both $X X$ and $X O$. This could be a secondary consequence of germ-line abnormality, but this explanation is unlikely because wild-type $X O$ animals whose germ cells have been diminished by laser ablation do not exhibit abnormalities of this type (Kimble and White 1981). The requirement for tra-1 activity is not absolute, because many mutant males have a functional male somatic gonad, although many do not. The variability of the somatic gonad in these mutants stands in contrast to the invariant nongonadal phenotype.

With regard to the germ-line phenotype, tra-1 function again appears to be complex. Both $g f$ and some $1 f$ alleles (classes A2 and A3, at least) can lead to the production of oocytes in an $X O$ animal (where they are never normally found). As suggested previously (Doniach and Hodgkin 1984), tra-1 activity does not seem to be essential for the formation of either sperm or oocytes, but it has an important role in determining which gametes are made. It is striking that all tra-1(gf) alleles isolated so far eliminate spermatogenesis from $X X$ animals, although most (17/22) were obtained by selections strongly biased toward maintenance of spermatogenesis. For this reason, it 
is likely that tra-1 can exert a negative effect on spermatogenesis. This conclusion is reinforced by the observation that $f e m-3(g f)$ mutations, which lead to constitutive spermatogenesis in mutant $X X$ hermaphrodites (Barton et al. 1987), can be suppressed by tra-1 (gf) mutations (K. Barton and J. Kimble, pers. commun.; J. Hodgkin, unpubl.). However, spermatogenesis is also reduced in tra-1(lf) mutants. A low level of spermatogenesis is to be expected in tra-1(lf) $X X$ mutants, because fem activity is limited in an $X X$ animal and spermatogenesis appears to be absolutely dependent on fem activity (Doniach and Hodgkin 1984). A low level is not expected in tra-1(If) XO mutants, because the fem genes should be fully active, promoting abundant spermatogenesis. For class Al alleles, it is conceivable that the low level of spermatogenesis is a secondary consequence of abnormality in the somatic gonad, but this seems less likely for class A3 alleles, because the somatic gonad in these mutants appears to be normal. One consistent correlation is that tra-1(lf) mutants with large, well-formed male gonads usually contain apparent oocytes. Formation of oocytes in class A1 mutants may therefore be inhibited because of the small size or abnormality of the gonad. A possible rationalization of all these observations is that both the somatic and the germ-line components of the male gonad are partly dependent on a low level of tra-1 activity. In the partial absence of tra-1 activity, reduced numbers of both sperm and oocytes are made; in the complete absence of tra-1 activity, somatic gonadal abnormalities prevent formation of any late gametes, so only sperm are made.

The finding that tra-1 has functions in both sexes of $C$. elegans (major function in $X X$; minor function in $X O$ ) is striking in view of the fact that the same is true for several of the major sex-determining loci of Drosophila: the $S x l, t r a-2$, and $d s x$ genes all have functions in both male and female flies (Baker and Belote 1983; Maine et al. 1985).

From these data, it would appear that the tra-1 gene has most of the genetic properties expected of a "complex gene," in the sense of Drosophila loci such as the Bithorax Complex, the scute-achaete complex, the $S x I$ and $d s x$ genes, and so forth. Whether other features of Drosophila complex genes, such as large physical size and complex transcription pattern, are also found in tra-1 must await molecular characterization of the locus.

\section{Materials and methods \\ General}

Standard methods of culture, manipulation, and examination were used (Brenner 1974; Doniach and Hodgkin 1984). Experiments were carried out at $20^{\circ} \mathrm{C}$ unless otherwise noted. Gamete types were determined by visual inspection using Nomarski microscopy: sperm are small, round cells with compact dotlike nuclei; oocytes are very large, round, or oblong cells with large nuclei and often with conspicuous nucleoli. Gonad and gamete examination was carried out on young adult males, 12-36 hr after the last larval molt. Between 20 and 40 individuals of each genotype were examined for the data in Figure 5.
Yolk protein synthesis was examined by visual inspection, using Nomarski microscopy, or by gel electrophoresis of ${ }^{35} \mathrm{~S}-\mathrm{la}-$ beled whole adult worms, using the procedures of Kimble and Sharrock (1983).

\section{Strains}

The following mutations were used: LGII, tra-2(e1209); LGIII, unc-32(e189), unc-47(e307), unc-49(e382), vab-7(e1562), dpy-18(e499), sup-1(e1955); LGIV, unc-17(e245), lin-3(e1417), dpy-20(e2017), dpy-26(n199), tra-3(e1107,e1767); LGV, him-5(e1490), egl-41(e2055), dpy-21(e428); LGX, dpy-7(e88), unc-18(e81), sup-7(st5), lon-2(e678). Alleles of tra-1 are listed in Tables 1 and 2. The sup-1 mutation is a dominant suppressor of unc-17(e245) but has no other phenotype (J. Hodgkin, unpubl.).

\section{Isolation and maintenance of tra-1(lf) alleles}

Seven tra-1 alleles (e440, e1076, e1099, e1488, e1516, e1928, e1929) have been recovered during routine mutant screens, by S. Brenner, P. Anderson, E. Hedgecock, and J. Hodgkin. One of these (e1488) was isolated after acetaldehyde mutagenesis, and the remainder, after EMS mutagenesis.

Another tra-1 allele, e1728, was isolated as an extragenic suppressor of her-1 (Hodgkin 1980). Eight more were isolated, as described below. All have been maintained as stocks of genotype tra-1; eDp6, or $v a b-7+d p y-18 /+\operatorname{tra}-1+$.

\section{A general screen for autosomal masculinizing mutations}

A strain CB3609 of genotype $d p y-7+1+$ unc- $18 X$ was used for this screen. The two sex-linked markers $d p y-7$ and unc-18 are linked tightly to each other (recombination distance, $\sim 0.4$ map units), so that hermaphrodites of this genotype will segregate hermaphrodite self-progeny in the ratio 2 wild-type: 1 dumpy : 1 uncoordinated. The occasional $X O$ self-progeny will be either dumpy or uncoordinated, and wild-type (non-Dpy, non-Unc) males are never seen in the population. Of strain CB3609, 213 hermaphrodites were mutagenized with EMS at L4 stage and placed on separate large $(9-\mathrm{cm})$ NGM plates (Brenner 1974). In the $F_{1}$ generation, all produced the expected three classes of hermaphrodite progeny. Brood counts on animals picked in parallel showed that each mutagenized $P_{o}$ animal produced about 100 (mean 97) wild-type $F_{1}$ hermaphrodites, indicating that a total of 43,000 haploid autosomal genomes were screened. No $F_{1}$ wild-type male progeny were seen, but in the $F_{2}$ generation, $51 / 213$ plates were found to produce several wildtype males or intersexes, which were presumably transformed $X X$ animals. In $5 / 51$ cases, these animals were fertile $X X$ males, so the mutation responsible was recovered easily by mating the males with marked hermaphrodites. All five were tra-1 alleles $(e 1729, e 1730, e 1774, e 1781, e 1783)$. In other cases, the males were not fertile, so each mutation had to be isolated by picking groups of hermaphrodites in smaller and smaller numbers in successive generations, until a single $t r a /+$ hermaphrodite could be identified. This somewhat laborious procedure was only used for $8 / 46$ cases, because most $(41 / 46)$ of the infertile males had the distinctive phenotype characteristic of tra-2 $X X$ males. To ascertain that these were tra-2 mutants, $6 / 41$ were isolated: $5 / 6$ were indeed $t r a-2$, and the sixth was a tra-1 allele (e1766). After outcrossing, the phenotype of $e 1766$ resembled tra-1 rather than tra-2, so the original stock probably carried modifier mutations that made it resemble a Tra- 2 phenotype. An additional two nonmating tra-1 mutants were recovered (e1732 and e1764), both of which had unusual phenotypes. Three further plates carried apparent tra-1 mutants, but these 
were not recovered. Thus, out of 43,000 mutagenized genomes screened, 8-11 tra-1 visible alleles were induced (8 recovered), and an estimated 40 tra- 2 alleles were induced $(5$ recovered), giving forward mutation frequencies of $2 \times 10^{-4}$ for tra-1 and $10^{-3}$ for tra-2. The $\mathrm{F}_{3}$ population was screened cursorily for wild-type males on the 162 plates that segregated no $F_{2}$ males, in order to detect maternal-effect tra mutations. Each plate carried about $10^{5}$ starved $F_{3}$ animals, so screening was not efficient. One maternal-effect mutant was recovered, e1767; this proved to be an allele of tra-3 (Hodgkin 1985, 1986).

\section{Isolation of tra-1(gf) alleles}

The first tra-1(gf) allele, e1575, was isolated during a general screen for mutations transforming $X O$ animals into fertile hermaphrodites or females (Hodgkin 1980). Thirteen additional mutations were isolated as extragenic suppressors of tra-3, these alleles being $e 1951, e 1953, e 1961, e 1962, e 1997, e 1993$ e1998, e2009, e2065, e2067, e2069, e2071, and e2097 (Hodgkin 1986). Reversion experiments on other weakly masculinizing mutations, tra-2(e1209) and egl-41(e2055), have also yielded tra-1(gf) mutations: alleles $e 2198$ and $e 2271$ from the former, and $e 2109$ from the latter (J. Hodgkin, unpubl.; T. Doniach unpubl.). Screens for dominant $X X$ feminizing mutations have yielded five alleles: $e 1937, e 1946, e 2013, e 2014$, and $e 2098$ (Doniach 1986, and unpubl.). These studies (Doniach 1986; Hodgkin 1986) suggest that the frequency of tra-1(gf) mutations is $2 \times 10^{-5}$ or lower, which is about 10 -fold less frequent than tra-1(lf) mutations.

Most tra-1(gf) alleles were propagated using strains homozygous for tra-3(e1767): tra-1(gf)/ +; tra-3XX animals are either hermaphrodite (for weak alleles) or female (for strong alleles), so strains can be propagated by selfing, in the first case, or crossing with tra-3(e1767) XO males, in the second case.

The homozygous tra-1(e1575) XO animal shown in Figure $8 \mathrm{~A}$ was generated by selfing an animal of genotype $e 1575 /+$; dpy-26 XO (Hodgkin 1983c).

\section{Reversion of a tra-l(gf) allele}

The allele $e 1575$ was used. A strain consisting of tra-1(e1575)/ + ; tra-3(e1107) females and tra-3 males $(X O)$ and intersexes $(X X)$ was constructed and propagated as an obligate male/female strain. A population $\left(\sim 10^{4}\right.$ individuals, mixed ages) was mutagenized with $0.05 \mathrm{M}$ EMS, grown for one generation, and plated on 10 large NGM plates containing tetramisole (Brenner 1974). This drug prevents movement but not growth and reproduction by self-fertilization, so self-fertile animals were selected. Of 10 plates, one yielded self-fertile hermaphrodites, one of which was used to found a stock, self-fertile hermaphrodite genotype tra-1(e1816e1575)/ + ;ra-3(e1107) XX. Further growth at low temperature yielded a homozygous stock (CB3373), tra-1(e1816e1575); tra-3, which is weakly self-fertile at $15^{\circ} \mathrm{C}$ almost completely female at $20^{\circ} \mathrm{C}$, and completely female at $25^{\circ} \mathrm{C}$. The partial revertant allele, $e 1816 e 1575$, still has a strong dominant feminizing effect: At $25^{\circ} \mathrm{C} e 1816 e 1575 /++X X$ animals are female, and $X O$ animals are sterile intersexes (see Fig. $8 \mathrm{~B}, \mathrm{C})$. The CB3373 stock is only self-fertile because of the presence of the tra-3 mutation (i.e., the tra-1 $(\mathrm{gf})$ and the tra-3 mutations are mutually suppressive at $\left.15^{\circ} \mathrm{C}\right)$. Consequently, intragenic revertants of tra-1(e1816e1575) were selected easily by crossing mutagenized $\mathrm{CB} 3373 \mathrm{XX}$ animals with wild-type males and shifting to $25^{\circ} \mathrm{C}$. All $\mathrm{F}_{1}$ cross progeny must be tra-1 $(g f) /+$; tra-3/ + , which are either female $(X X)$ or intersexual $(X O) . F_{2}$ progeny are only obtained if the $F_{1}$ population contains hermaphrodites or males; these can be generated by an intragenic event within tra-1 or by an extragenic suppressor. A total
$F_{1}$ population of approximately $1.5 \times 10^{5}$ individuals was screened, derived from about $1600 \mathrm{P}_{\mathrm{o}}$ females mutagenized with 0.05 or $0.075 \mathrm{M}$ EMS, and crossed with wild-type males in sets of three or four females per small plate. Twenty-eight independent tra-1(tr) alleles were recovered, usually as $X X$ hermaphrodites of genotype tra-1(tr)/ + . Apparent males [tra-1(tr)/ $+X O]$ were seen on a number of plates but were often unable to sire progeny. Two tra-2 recessive alleles were also recovered, because tra- 2 null alleles have weak dominant masculinizing effects (Trent et al. 1983), and, therefore, tra-2/+; tra-1(e1816e1575)/ $+X X$ animals are slightly self-fertile. Smaller-scale reversion CB3373 experiments were also carried out, using either diepoxyoctane (Anderson and Brenner 1982), ultraviolet light (Greenwald and Horvitz 1980), or X-rays [8000 roentgen (Herman et al. 1976)] as a mutagen. Only the last yielded any revertants: The recessive lethal $e 1855$ was recovered as an $\mathrm{e} 1855 /+$; tra $-3 /+X O$ male, out of about $2 \times 10^{4} \mathrm{~F}_{1}$ progeny. Homozygotes for e1855 die as late embryos, but e1855/e1099 XX heterozygotes are viable healthy transformed males. Attempts to separate the lethality from the tra-1 mutation were made: A total of 136 Unc and 132 Dpy recombinants were picked from a heterozygote unc- $47+d p y-18 /+e 1855+$, but none of these segregated $F_{2}$ Unc Tra or Dpy Tra progeny. Recombination between these flanking markers, unc- 47 and $d p y-18$, was slightly but significantly reduced in this heterozygote (linkage 4.5 map units as compared to 6.2 map units in a control heterozygote unc-47 $d p y-18 /++1$. Subsequently $\mathrm{H}$. Schnabel (pers. commun.) has found that $e 1855$ fails to complement an embryonic lethal mutation, pha-1(e2123), which maps between vab-7 and tra-1 and complements these two markers. It is likely that $e 1855$ is a small deficiency, but it is also possible that it is a small inversion.

\section{Suppression tests}

Recessive tra-1 alleles were tested for amber-suppressibility by selfing hermaphrodites of genotype unc-32 tra-1/ + + sup- $7 /+$ and examining tail phenotypes of Unc animals. In the absence of suppression, most $(85 \%)$ of the Unc animals exhibit a Tra phenotype, and a few $(15 \%)$ are completely non-Tra recombinants (unc-32+/unc-32 tra-1). The appearance of many nonTra Unc progeny, or intersexual Unc progeny, indicated suppression. All recessive tra-1 alleles in Table 1 have been tested, except for e1928, e1929, e1837tr, e1840tr, e1843tr, and e1853tr. gf tra-1 alleles were tested by crossing tra-1(gf); tra-3(e1767) $X X$ females with $d p y-20 /+$; sup-7/0 males and crossing progeny $X X$ females with more of the same males; $X O$ progeny were examined, in order to see if any had a less feminized phenotype than control tra-1(gf)/ $+X O$ progeny. Also, suppressed dumpy $X X$ progeny could usually be recognized (Hodgkin 1985); the appearance of suppressed dumpy female $X X$ animals was taken to indicate lack of suppression. Eleven tra-1(gf) alleles were tested, and none showed any sign of suppressibility (alleles e1575, e1951, e1953, e1961, e1962, e1993, e2013, e2014, e2071, e2109, and e2198).

\section{Construction of tra-1 XO animals}

Wild-type males, tra-1 $X X$ males, and tra-1 XO males have almost identical phenotypes by dissecting microscope; therefore, marked tra-1 XO males were generated by the following crosses: unc-32 vab-7 tra-1/unc-32 vab-7 + dpy-18 hermaphrodites crossed with $d p y-18 \mathrm{XO}$ males, yielding non-Dpy nonUnc males of genotype unc-32 vab-7 tra-1 $+1+++$ dpy- 18 . These were crossed with hermaphrodites of genotype $v a b-7+d p y-18 /+$ tra-1 dpy-18; lon-2 X. Non-Vab, non-Dpy 
Lon progeny of this cross will be almost all of genotype ++ tra-1 dpy-18/unc-32 vab-7 tra-1; lon-2/0, apart from rare recombinants (which may account for most or all of the rare fully male animals seen). XO males of tra-1(e1099) generated by this means were sometimes unusually thin, but $e 1099 \mathrm{XX}$ males were not thin, nor were $X O$ males of $e 1781$ and $e 1835 t r$. The lon-2 mutation leads to an abnormally long body, but does not significantly alter gonad morphology in control lon-2/0 males (Figs. 4E and 5J). Other markers are recessive.

\section{Acknowledgments}

I thank many colleagues for providing strains, advice, and suggestions. I am particularly grateful to John Sulston for detailed cellular examination of some of the tra-1 mutants; to Tabitha Doniach, Michael Shen, and Judith Kimble for extensive discussions; and to Tim Schedl for discovering some of the gonadal complexity of tra-1 mutants.

\section{References}

Ambros, V. and H.R. Horvitz. 1987. The lin-14 gene controls developmental timing. Genes Dev. 1: 398-414.

Anderson, P. and S. Brenner. 1984. A selection for myosin heavy chain mutants in the nematode Caenorhabditis elegans. Proc. Natl. Acad. Sci. 81: 4470-4474.

Baker, B.S. and J. Belote. 1983. Sex determination and dosage compensation in Drosophila melanogaster. Ann. Rev. Genet. 17: 345-393.

Barton, M.K., T. Schedl, and J. Kimble. 1987. Gain-of-function mutations of fem-3: A sex-determination gene in Caenorhabditis elegans. Genetics 115: 107.

Brenner, S. 1974. The genetics of C. elegans. Genetics 77: 71.

Doniach, T. 1986. Activity of the sex-determining gene tra- 2 is modulated to allow spermatogenesis in the $C$. elegans hermaphrodite. Genetics 114: 53-76.

Doniach, T. and J. Hodgkin. 1984. A sex-determining gene, fem-1, required for both male and hermaphrodite development in C. elegans. Dev. Biol. 106: 223-235.

Greenwald, I.S. and H.R. Horvitz. 1980. unc-93(e1500): A behavioral mutant of $C$. elegans that defines a gene with a wildtype null phenotype. Genetics 96: 147.

Greenwald, I.S., P. Sternberg, and H.R. Horvitz. 1983. The lin-12 locus specifies cell fates in C. elegans. Cell 35: 435444.

Greenwald, I., A. Coulson, J. Sulston, and J. Priess. 1987. Correlation of the physical and genetic maps in the lin-12 region of Caenorhabditis elegans. Nucleic Acids Res. 15: 22952307.

Herman, R.K., D.G. Albertson, and S. Brenner. 1976. Chromosome rearrangements in C. elegans. Genetics 83: 91-105.

Hodgkin, J. 1980. More sex determination mutants of Caenorhabditis elegans. Genetics 96: 649-664.

-1983a. Male phenotypes and mating efficiency in Caenorhabditis elegans. Genetics 103: 43-64.

.1983b. Two types of sex determination in a nematode. Nature 304: 267-268.

-1983c. X chromosome dosage and gene expression in $C$. elegans: Two unusual dumpy genes. Mol. Gen. Genet. 192: $452-458$.

.1984. Switch genes and sex determination in the nematode C. elegans. I. Emb. Exp. Morphol. 83 (Suppl.): 103.

.1985. Novel nematode amber suppressors. Genetics 111: $287-310$.

-1986. Sex determination in the nematode C. elegans: Analysis of tra-3 suppressors and characterization of fem genes. Genetics 114: 15-52.
Hodgkin, J.A. and S. Brenner. 1977. Mutations causing transformation of the sexual phenotype in the nematode Caenorhabditis elegans. Genetics 86: 275-287.

Hodgkin, J., T. Doniach, and M. Shen. 1986. The sex determination pathway in the nematode Caenorhabditis elegans: Variations on a theme. Cold Spring Harbor Symp. Quant. Biol. 50: 585-593.

Hodgkin, J., H.R. Horvitz, and S. Brenner. 1979. Nondisjunction mutants of the nematode Caenorhabditis elegans. Genetics 91: 67-94.

Kimble, J. 1981. Alterations in cell lineage following laser ablation of cells in the somatic gonad of Caenorhabditis elegans. Dev. Biol. 87: 286-300.

Kimble, J. and D. Hirsh. 1979. The postembryonic cell lineages of the hermaphrodite and male gonads in Caenorhabditis elegans. Dev. Biol. 70: 396-417.

Kimble, J.E. and W.J. Sharrock. 1983. Tissue-specific synthesis of yolk proteins in C. elegans. Dev. Biol. 96: 189-196.

Kimble, J.E. and J.G. White. 1981. On the control of germ cell development in Caenorhabditis elegans. Dev. Biol. 96: $208-219$.

Madl, J.E. and R.K. Herman. 1979. Polyploids and sex determination in Caenorhabditis elegans. Genetics 93: 393-402.

Maine, E.M., H.K. Salz, P. Schedl, and T.W. Cline. 1985. Sexlethal, a link between sex determination and sexual differentiation in Drosophila melanogaster. Cold Spring Harbor Symp. Quant. Biol. 50: 595-604.

Moerman, D.G. and D.L. Baillie. 1979. Genetic organization in Caenorhabditis elegans: Fine structure analysis of the unc-22 gene. Genetics 91: 95-103.

Sternberg, P.W. and H.R. Horvitz. 1984. The genetic control of cell lineage during nematode development. Ann. Rev. Genet. 18: 489-524.

Sulston, J.E., D.G. Albertson, and J.N. Thomson. 1980. The Caenorhabditis elegans male: Postembryonic development of nongonadal structures. Dev. Biol. 78: 542 .

Sulston, J.E. and H.R. Horvitz. 1977. Post-embryonic cell lineages of the nematode, C. elegans. Dev. Biol. 56: 110

Sulston, J.E. and J.G. White. 1980. Regulation and cell anatomy during postembryonic development of C. elegans. Dev. Biol. 78: 577.

Swanson, M.M., M.L. Edgley, and D.L. Riddle. 1984. The nematode Caenorhabditis elegans. In Genetic maps 1984 (ed. S.J. O'Brien), Cold Spring Harbor Laboratory, New York.

Waterston, R.H. 1981. A second informational suppressor, sup-7, in C. elegans. Genetics 97: 307-325.

Waterston, R.H., K.C. Smith, and D.G. Moerman. 1982. Genetic fine structure analysis of the myosin heavy chain gene unc-54 of Caenorhabditis elegans. J. Mol. Biol. 185: 1-15. 


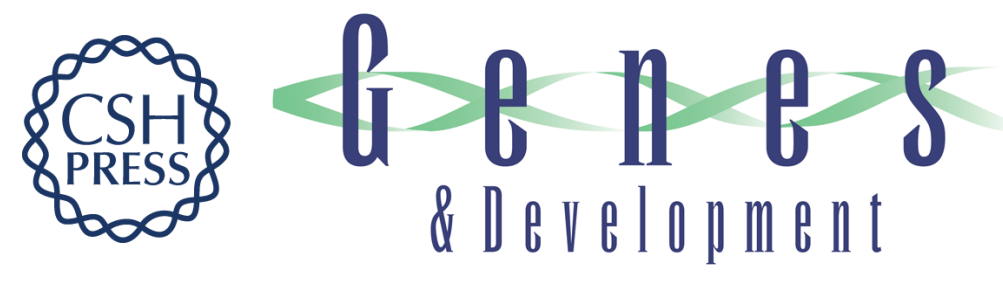

\section{A genetic analysis of the sex-determining gene, tra-1, in the nematode Caenorhabditis elegans.}

J Hodgkin

Genes Dev. 1987, 1:

Access the most recent version at doi:10.1101/gad.1.7.731

References This article cites 34 articles, 17 of which can be accessed free at: http://genesdev.cshlp.org/content/1/7/731.full.html\#ref-list-1

License

Email Alerting

Receive free email alerts when new articles cite this article - sign up in the box at the top Service right corner of the article or click here.

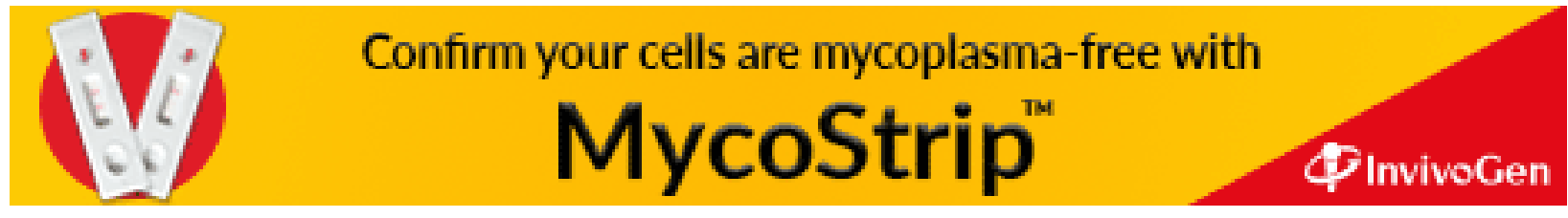

